\title{
Core outcome set for infant gastroesophageal reflux disease
}

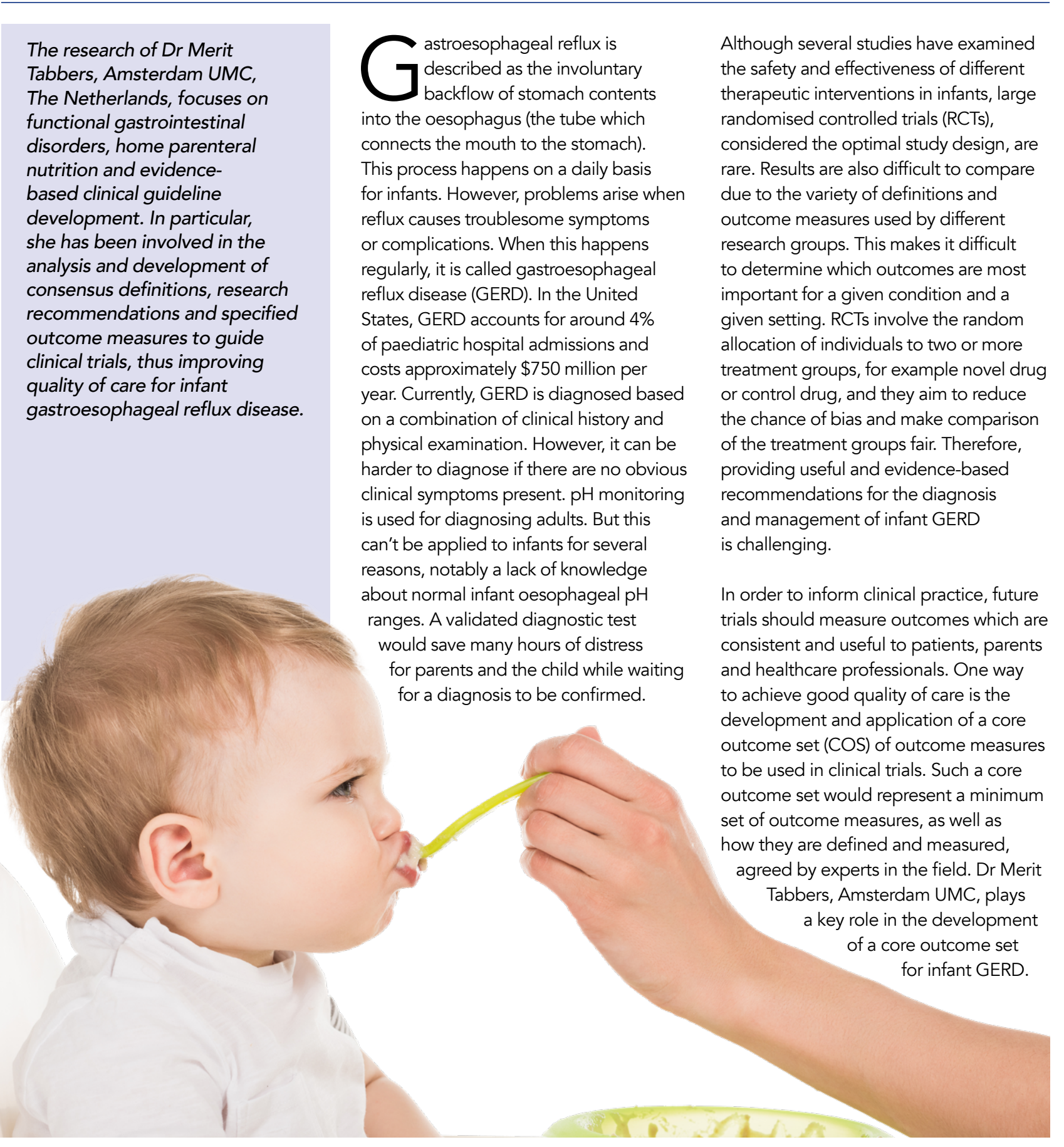




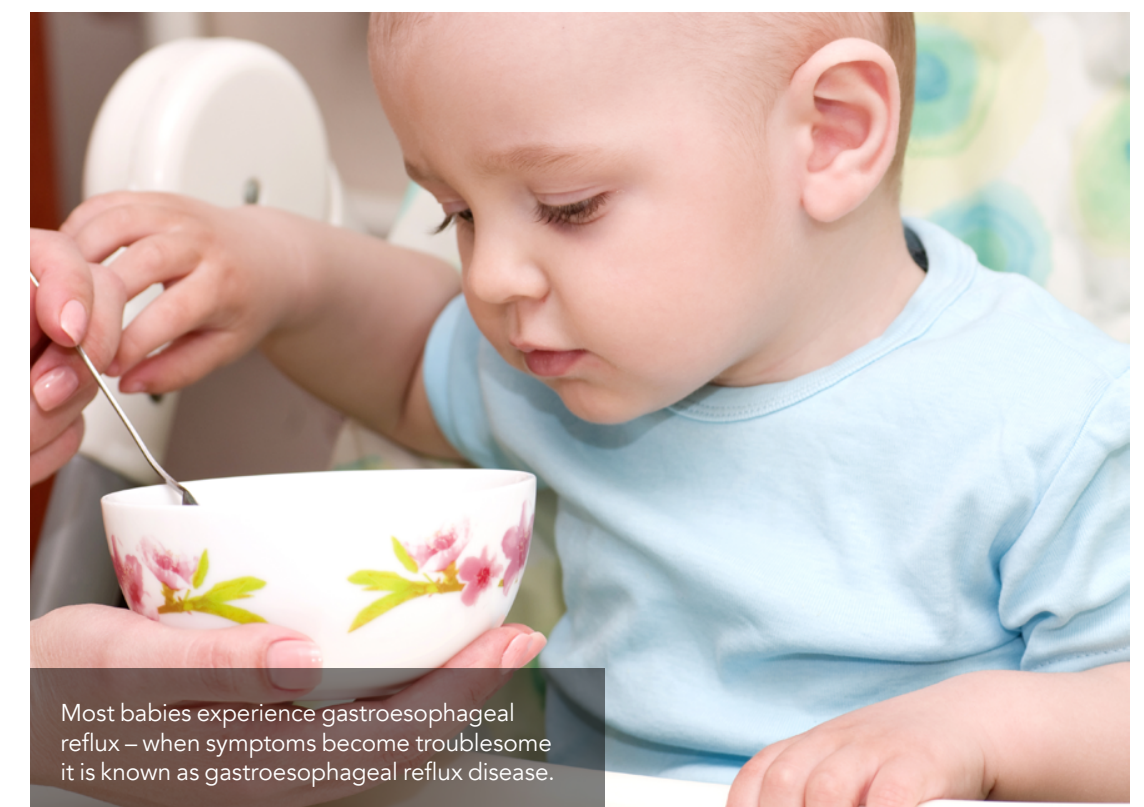

DEVELOPING CONSENSUS

Leading on from this, and informed

a cOS for infant GERD. Dr Tabbers

outcomes, such as crying and feeding difficulties, clinical outcomes, such as

of medication use, and adverse events.

The COS developed by Dr Tabbers and colleagues has the potential to improve quality of care for infants with GERD.

worked alongside colleagues from around Europe and the United States to develop a nine-item $\mathrm{COS}$ which This tool has the potential to decrease the heterogeneity of future studies and ease comparability of study results. The

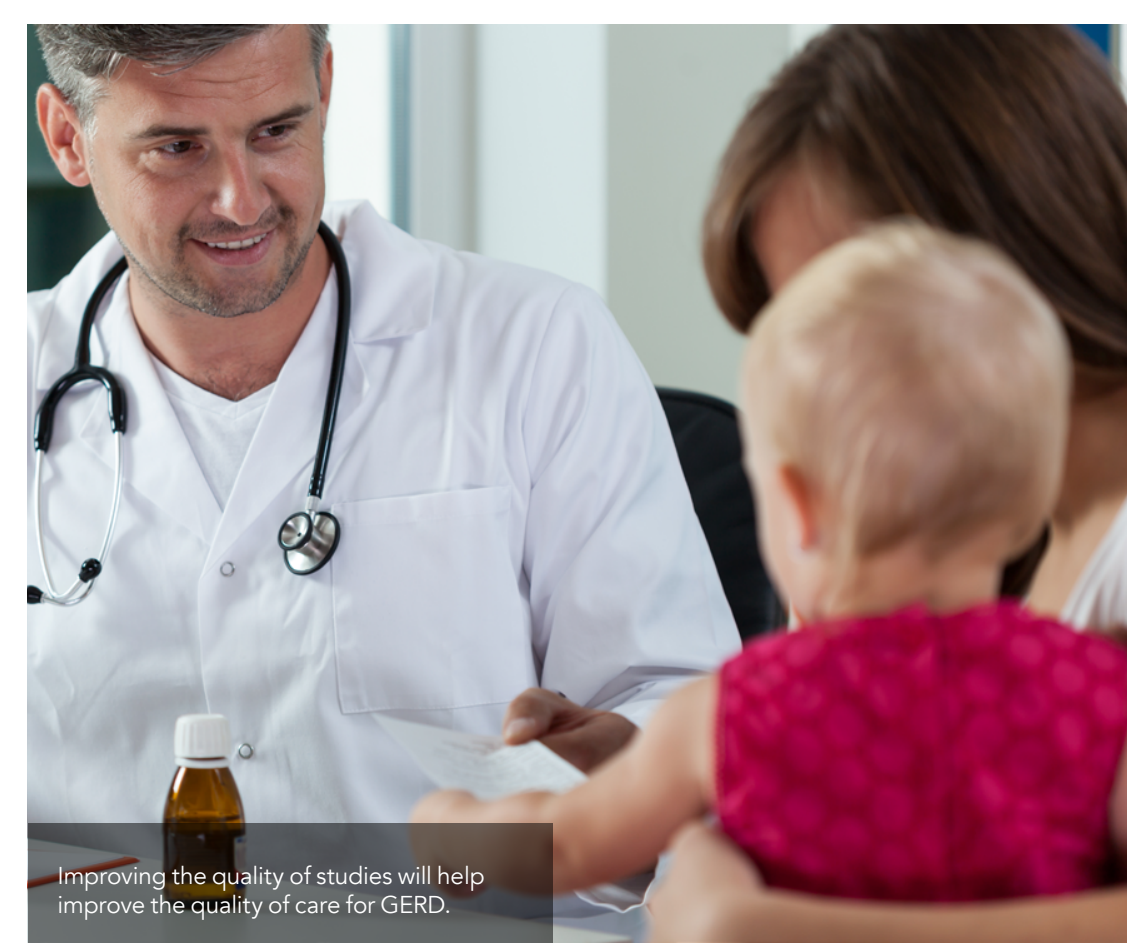

international Delphi study. This technique question sening multiple rounds of to a group of experts, with the aim of generating consensus opinions about topic. Clinical practice guidelines for paediatric reflux were published a year earlier, a project also led by Dr Tabbers, and were used to guide the COS. Indeed, without consensus definitions and outcome measures, it is challenging to answer many of these questions due to conflicting studies and poor-quality evidence, further highlighting the need for more rigorous studies for infant GERD.

Instead of developing a set of outcome standards, these clinical guidelines, publshed jointly by the North American and European research societies, aimed evidence to answer eight clinical research questions. The questions covered all aspects of reflux and GERD, ranging from definition and 'red flag' symptoms, to evidence for pharmacologic, nonpharmacologic and surgical treatment options and finally considering evaluation and prognosis of GERD.

IMPACT AND CHALLENGES The COS developed by Dr Tabbers and colleagues has the potential to improve quality of care for infants with GERD. However, the COS is dynamic and will require review and adjustment as more studies are done. Furthermore, different on disease severity study design and the isease severity, study design and the heterogeneous definitions of infant GERD and the lack of a gold standard diagnostic test, the next challenge may be to develop clear consensus for defining GERD in different patient populations. These challenges are not limited to infant GERD alone, but represent common issues with clinical trials for all diseases, especially when research teams are limited by resources, funding or personne.

Despite this, development of a set of defined outcome measures which must be adhered to is a major step towards improving the quality of studies done around infant GERD, and therefore, an important advancement in quality

\section{Behind the Research}

\section{Dr Merit Tabbers}

E: m.m.tabbers@amsterdamumc.nl T: +31 205663053

\section{Research Objectives}

Dr Tabbers' work looks at gastroesophogeal reflux disease in infants - a condition where stomach contents flow into the oesophagus and cause irritation.

\section{Detail}

Meibergdreef 9

Me DD Amsterdam

Since 2007 Merit Tabbers has been a staff member in the department of Paediatric gastroenterology and nutrition at the Emma Children's Hospita/Amsterdam UMC. She is author of different national and international guidelines. The focus of her current clinical and research work is evidence-based guideline development, functiona gastrointestinal disorders and home parenteral nutrition.

Funding

Networking grant from ESPGHAN society

Collaborators

All health care professionals and parents who have been ivolved in $\mathrm{COS}$ developmen

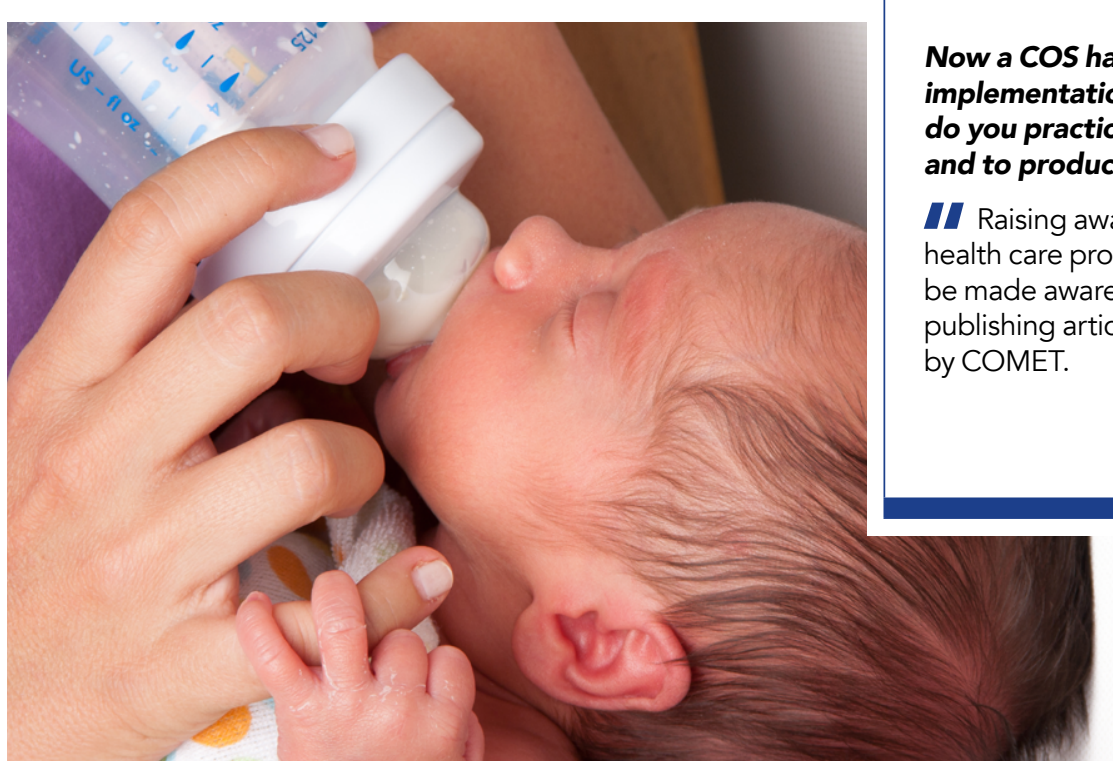

References

Kirkham, J.J., Davis, K., Altman, D.G., Blazeby, J.M., Outcome Set-STAndards for Development: The COS-STAD recommendations. PLoS Med. 14(11): e1002447. doi.

org/10.1371/journal.pmed.1002447

Singendonk, M.M.J., Brink, A.J., Steutel, N.F., van EttenM.M. (2017). Variations in Definitions and Outcome Measures in Gastroesophageal Reflux Disease: A Systematic Review.

Rosen, R., Vandenplas, Y., Singendonk, M., Cabana, M.', Staiano, A. Thapar, N., Tipnis, N. \& Tabbers, M. (2018). Pediatric Gastroesophageal Reflux Clinical Practice Guidelines: Joint Recommendations of the North American Society for Pediatric Gastroenterology, Pediatric Gastroenterology. Hepatology and Nutiety for Pediatr Gastroenterol Nutr, 66(3), 516-554. doi:10.1097 mpg.0000000000001889

Singendonk, M. M. J., Rexwinkel, R., Steutel, N. F., Gottrand F, McCall, L., Orsagh-Yentis, D. K., Rosen, R., Strisciuglio, C M. (2019). Development of a Core Outcome Set for Infant Gastroesophageal Reflux Disease. J Pediatr Gastroenterol
Nutr, 68(5), 655-661. doi:10.1097/mpg.000000000002245

\section{Personal Response}

been established, what does the

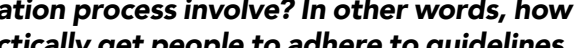
tuce high-quality, comparable studies?

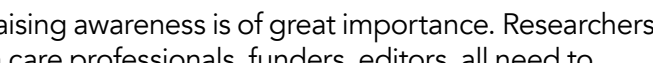
政 ishing articles and we will officially register the COS

UU Emma Children's Hospital 